HEPHY-PUB 937/14, CUQM 151

\title{
Schrödinger models for solutions of the Bethe-Salpeter equation in Minkowski space. II. Fermionic bound-state constituents
}

\author{
Richard L. Hall ${ }^{1, *}$ and Wolfgang Lucha, \\ ${ }^{1}$ Department of Mathematics and Statistics, Concordia University, \\ 1455 de Maisonneuve Boulevard West, Montréal, Québec, Canada H3G 1M8 \\ ${ }^{2}$ Institute for High Energy Physics, Austrian Academy of Sciences, \\ Nikolsdorfergasse 18, A-1050 Vienna, Austria
}

\begin{abstract}
In view of the obstacles encountered in any attempts to solve the Minkowski-space Bethe-Salpeter equation for bound states of two fermions, we study the possibility to model the bound-state features, at least at a qualitative level, by a Schrödinger description. Such a nonrelativistic potential model can be constructed by applying, to any given Bethe-Salpeter spectral data, 'geometric spectral inversion' in its recently extended form, which tolerates also singular potentials. This leads to the adaptation of explicit models that provide an overview accounting for the Bethe-Salpeter formalism's complexities.

PACS numbers: 11.10.St, 03.65.Pm, 03.65.Ge.

Keywords: Bethe-Salpeter formalism, nonrelativistic potential model, geometric spectral inversion, Coulomb Schrödinger model, Hulthén Schrödinger model
\end{abstract}

\section{INTRODUCTION: MOTIVATION AND INCENTIVE}

The Bethe-Salpeter formalism offers a description of bound states consistent with all the requirements of relativistic quantum field theory [1 3] : bound states with all their properties, such as their masses or the distribution of the relative momenta of their constituents (encoded in suitably defined amplitudes playing the role of a kind of wave functions) arise as solutions of appropriate equations of motion, called the (homogeneous) Bethe-Salpeter (BS) equation in the case of bound states composed of two constituents or the (homogeneous) Faddeev equation for three-particle systems. In these equations, the underlying dynamics enter via the full propagators of the constituents and their interactions kernels Green functions which, at least in principle, may be deduced from the infinite tower of corresponding Dyson-Schwinger equations. In practice, we are limited to adequate truncations of this tower and the tight bounds of perturbation theory.

However, attempts to obtain solutions to the BS equation in Minkowski space, characterized by the pseudo-Euclidean space-time metric tensor $g_{\mu \nu}=\operatorname{diag}(+1,-1,-1,-1)$, have to face serious obstacles in form of singularities induced by propagators or interaction kernels. The usual remedy is to trust in analytic continuation and Cauchy's integral theorem and to formulate the BS equation in Euclidean space, with metric $g_{\mu \nu}=\delta_{\mu \nu}$. However, even if the two approaches yield the same bound-state masses, predictions based on the amplitudes describing the constituents' motion differ drastically.

In view of this, we recently proposed [4] to construct, by spectral inversion of the bound-state energies, approximately equivalent Schrödinger models, and applied [4] this idea, as a kind of feasibility study, to Minkowski-space BS results for bound states of two scalar particles. Here, we extend this analysis to the case of higher relevance for physics: fermionic bound-states constituents. Couplings of fermions to bosons distinguish between bosons of different Lorentz nature. We use as input BS findings from exchange of a single scalar, pseudoscalar, or massless vector boson reported in Refs. [5-7], and invert the data by our generalization to singular potentials [8] of a previously formulated inversion technique 9 14]. We may get a first idea of what to expect by inspecting the BS formalism's nonrelativistic limit; see, e.g., Refs. [15 18].

The outline of this paper is as follows. In Sec. [II], we recall, merely to the extent required for the present investigation, the principal features of our previously constructed geometric spectral inversion theory [4, 8]. In order to prepare the grounds for the applications of this approach to the energy levels of fermion-(anti-)fermion bound states resulting from the (Minkowski-space) BS formalism, we collect, in Sec. [II], the binding-energy predictions of Refs. [5 [7]. In Sec. [IV] we subject these mass spectra to the inversion procedure. The insights gained by these efforts finally motivate us to search, in Sec. $\nabla$ for analytic models, based on shifted-Coulomb or Hulthén potentials, rather accurately reproducing the data.

\footnotetext{
*Electronic address: rhall@mathstat.concordia.ca

${ }^{\dagger}$ Electronic address: wolfgang.lucha@oeaw.ac.at
} 


\section{GEOMETRIC SPECTRAL INVERSION}

Let us start by sketching briefly the reasoning of Refs. [4, 8] leading to the spectral inversion algorithm and stating a uniqueness theorem. The underlying functional inversion was first introduced in Ref. [14]. We consider the discrete spectrum of a Schrödinger Hamiltonian operator

$$
H \equiv-\Delta+v f(r), \quad r \equiv\|\boldsymbol{r}\|, \quad \boldsymbol{r} \in \mathbb{R}^{3}
$$

where $f(r)$ is an attractive central-potential shape and $v>0$ is its coupling parameter. Assume that $f(r)$ is monotone non-decreasing and no more singular than the Coulomb potential $f(r)=-1 / r$. Then, the operator inequality [19, 20] $H \geq 1 /\left(4 r^{2}\right)+v f(r)$, along with a simple variational upper bound to $\langle H\rangle$, enables us to show that a discrete spectrum exists for sufficiently large coupling $v>v_{1}>0$. In particular, the ground-state energy $E$ may be written as a function $E=F(v)$. We are interested in the problem: can we reconstruct the potential shape $f(r)$ from the spectral data, given by, e.g., the ground-state curve $F(v)$ ? We call this type of reconstruction 'geometric spectral inversion'.

Discrete spectra of operators bounded from below can be characterized variationally [21]; their ground-state energy is

$$
F(v)=\inf _{\substack{\psi \in \mathcal{D}(H) \\\|\psi\|=1}}(\psi, H \psi),
$$

where $\mathcal{D}(H)$ is the domain of $H$. Define, for the ground state $\psi$, the kinetic potential $\bar{f}(s)$ associated with the potential $f(r)$ by a constrained minimization that keeps the mean kinetic energy $s \equiv\langle-\Delta\rangle$ constant:

$$
\bar{f}(s) \equiv \inf _{\substack{\psi \in \mathcal{D}(H) \\\|\psi\|=1 \\(\psi,-\Delta \psi)=s}}(\psi, f \psi) .
$$

A final minimization over $s$ allows us to recover the eigenvalue $F(v)$ of $H$ from $\bar{f}(s)$ :

$$
F(v)=\min _{s>0}[s+v \bar{f}(s)]
$$

The spectral function $F(v)$ turns out to be concave (i.e., $F^{\prime \prime}(v)<0$ ) and can been shown [9] to satisfy

$$
F^{\prime \prime}(v) \bar{f}^{\prime \prime}(s)=-\frac{1}{v^{3}}<0 .
$$

Hence, $F(v)$ and $\bar{f}(s)$ have opposite convexities and, moreover, are related by a Legendre transformation $\bar{f} \leftrightarrow F[22]$ :

$$
\begin{array}{lc}
\bar{f}(s)=F^{\prime}(v), & s=F(v)-v F^{\prime}(v), \\
\frac{1}{v}=-\bar{f}^{\prime}(s), & \frac{F(v)}{v}=\bar{f}(s)-s \bar{f}^{\prime}(s) .
\end{array}
$$

$F(v)$ is not necessarily monotone, but $\bar{f}(s)$ is monotone decreasing. By Eq. (6), in place of $s$ also the coupling, labelled $u$ for this purpose, may be used as minimization parameter.

A different formulation of this minimization is found if changing the kinetic-energy parameter from $s$ to $r$ itself, by inverting the (monotone) function $\bar{f}(s)$ to define the $K$-function

$$
K^{[f]}(r)=s=\left(\bar{f}^{-1} \circ f\right)(r)
$$

which exhibits invariance with respect to scale and shifts (with constants $A>0$ and $B$ ):

$$
K^{[A f+B]}(r)=K^{[f]}(r)
$$

In terms of $K$, Eq. (4) becomes

$$
F(v)=\min _{r>0}\left[K^{[f]}(r)+v f(r)\right] .
$$

Clearly, $K$ still depends on $f$, but Eq. (10) has $F(v)$ on one side and $f(r)$ on the other. By inversion of this relation, we may accomplish $F \rightarrow f$. To this end, we construct a sequence of approximate $K$-functions which do not depend on $f$.

Now, suppose that a $f(r)$ may be written as smooth transformation $f(r)=g(h(r))$ of a 'basis potential' $h(r)$. Then, the knowledge of the spectrum of $-\Delta+v h(r)$ may by exploited to study the spectrum of $-\Delta+v f(r)$. For definite 
convexity of the transformation function $g$, the kinetic-potential formalism immediately provides energy bounds. This follows from Jensen's inequality [23], which we rephrase, for our present goal, in terms of the kinetic-potential bounds

$$
g^{\prime \prime} \geq 0 \quad \Longrightarrow \quad \bar{f}(s) \geq g(\bar{h}(s)) ; \quad g^{\prime \prime} \leq 0 \quad \Longrightarrow \quad \bar{f}(s) \leq g(\bar{h}(s)) \text {. }
$$

For these, we write $\bar{f}(s) \approx g(\bar{h}(s))$, where the symbol $\approx$ is understood to indicate the appropriate inequality whenever $g$ has definite convexity. Expressed in terms of $K$-functions, the above results read

$$
K^{[f]}=\bar{f}^{-1} \circ f \approx(g \circ \bar{h})^{-1} \circ(g \circ h)=\bar{h}^{-1} \circ h=K^{[h]} .
$$

Thus, $K^{[f]} \approx K^{[h]}$ is the approximation sought; it no longer depends on $f$. The corresponding energy bounds become

$$
E=F(v) \approx \min _{s>0}[s+v g(\bar{h}(s))]=\min _{r>0}\left[K^{[h]}(r)+v f(r)\right] .
$$

For an eigenvalue $E$ of $H$ known as function $E=F(v)$ of the coupling $v>v_{1}$, the kinetic potential $\bar{f}(s)$ is found by inverting the Legendre transformation (6):

$$
F(v)=\min _{s>0}[s+v \bar{f}(s)] \quad \Longrightarrow \quad \bar{f}(s)=\max _{v>v_{1}}\left[\frac{F(v)}{v}-\frac{s}{v}\right] .
$$

Furthermore, we also have to invert the relation (10) between $F^{[n]}$ and $K^{[n]}$ :

$$
K(r)=\max _{v>v_{1}}[F(v)-v f(r)] .
$$

We implement the inversion procedure by starting from a suitably chosen seed potential shape, $f^{[0]}(r)$, from which we generate a sequence $\left\{f^{[n]}(r)\right\}_{n=0}^{\infty}$ of improving approximations to the potential. The idea behind this is to arrive at a map $g$ such that $g\left(f^{[n]}(r)\right)$ is close to $f(r)$ in the sense that the arising eigenvalue is close to our starting point $F(v)$. At each stage, the envelope approximation is used. At stage $n$, the best transformation $g^{[n]}$ is deduced by using the current potential approximation $f^{[n]}(r)$ as envelope basis. Thus, each step in the generation of the sequence $\left\{f^{[n]}(r)\right\}_{n=0}^{\infty}$ reads

$$
\bar{f}=g^{[n]} \circ \bar{f}^{[n]} \quad \Longrightarrow \quad g^{[n]}=\bar{f} \circ\left(\bar{f}^{[n]}\right)^{-1} \quad \Longrightarrow \quad f^{[n+1]}=g^{[n]} \circ f^{[n]}=\bar{f} \circ K^{[n]} .
$$

In more detail, the ultimate procedure of our inversion algorithm may be summarized symbolically in the following way:

$$
\begin{aligned}
f^{[n]}(r) & \longrightarrow \quad F^{[n]}(v) \\
& \longrightarrow \quad K^{[n]}(r)=\max _{u>v_{1}}\left[F^{[n]}(u)-u f^{[n]}(r)\right] \\
& \longrightarrow \quad f^{[n+1]}(r)=\max _{v>v_{1}}\left[\frac{F(v)}{v}-\frac{K^{[n]}(r)}{v}\right] .
\end{aligned}
$$

For the step $f^{[n]}(r) \longrightarrow F^{[n]}(v)$ need to know $E=F^{[n]}(v)$, which we get by solving $\left(-\Delta+v f^{[n]}\right) \psi=E \psi$ numerically.

The potential shape $f(r)$ is severely constrained by knowledge of $F(v)$. Consider a singular potential $f(r)$ of the form

$$
f(r)=\frac{g(r)}{r}
$$

where $g(0)<0, g^{\prime}(r) \geq 0$, and $g(r)$ is not constant. Examples of such singular shapes $f(r)$ are Yukawa, $g(r)=-e^{-a r}$, Hulthén, $g(r)=r /\left(e^{a r}-1\right)$, and linear-plus-Coulomb, $g(r)=-a+b r^{2}$, with $a, b>0$. For this class of potentials, we have proved [8] the following

Theorem: the potential $f(r)$ in $H=-\Delta+v f(r)$ is uniquely determined by its ground-state energy function $E=F(v)$.

\section{SPECTRAL DATA FROM MINKOWSKI-SPACE BETHE-SALPETER EQUATION FOR FERMIONIC BOUND-STATE CONSTITUENTS}

\section{A. Bethe-Salpeter bound-state energies as input data to spectral inversion}

In their discussion [5-7] of the homogeneous Bethe-Salpeter equation in Minkowski space, Carbonell and Karmanov consider bound states of two fermionic constituents of equal masses $m$, bound by exchanging between these constituents a single boson of mass $\mu$ and of either scalar, or pseudoscalar, or vector Lorentz nature. In Table 1 we reproduce, from Refs. [5 - 7], the five sets of associated binding energies, $E$, presently available in the literature, computed by numerical solution of the corresponding Minkowski-space Bethe-Salpeter equation, versus our coupling parameter, $v$, and, for the sake of later ease of reference, grasp the opportunity to label these data sets in a mnemonic way (last row of Table . $_{\text {. }}$ 
TABLE I: Couplings $v$ and binding energies $E$ arising from (Minkowski-space) Bethe-Salpeter equations describing bound states of fermionic constituents of mass $m=1$, computed for two-fermion systems bound by exchange of a single scalar or pseudoscalar boson of mass $\mu=0.15$ or $\mu=0.5$ and for fermion-antifermion systems bound by a single massless vector-boson exchange [5 7] $[4$ The numerical values of the binding energies $E$ have been computed for the choices $\Lambda=2$ for the vertex form-factor parameter $\Lambda$ in the vertex form factor $F(k)$ of Eq. (19) and $L=1.1$ for the 'mass' $L$ in the 'discontinuity-smoothing' factor $\eta(p, P)$ of Eq. (20).

\begin{tabular}{|c|c|c|c|c|c|}
\hline \multicolumn{5}{|c|}{$v$} & \multirow[t]{4}{*}{$E$} \\
\hline \multicolumn{4}{|c|}{ Fermion-Fermion Bound State } & \multirow{2}{*}{$\frac{\text { Fermion-Antifermion Bound State }}{\text { Vector-Boson Exchange }}$} & \\
\hline \multicolumn{2}{|c|}{ Scalar-Boson Exchange } & \multicolumn{2}{|c|}{ Pseudoscalar-Boson Exchange } & & \\
\hline$\mu=0.15$ & $\mu=0.50$ & $\mu=0.15$ & $\mu=0.50$ & $\mu=0$ & \\
\hline 0.6217 & 2.008 & 17.89 & 33.61 & 0.2598 & -0.01 \\
\hline 0.7998 & 2.347 & 18.53 & 34.23 & 0.3907 & -0.02 \\
\hline 0.9510 & 2.627 & 18.80 & 34.68 & 0.4984 & -0.03 \\
\hline 1.089 & 2.880 & 19.35 & 35.05 & 0.5934 & -0.04 \\
\hline 1.222 & 3.119 & 19.66 & 35.36 & 0.6802 & -0.05 \\
\hline 1.840 & 4.203 & 20.86 & 36.60 & 1.046 & -0.10 \\
\hline 3.049 & 6.227 & 22.51 & 38.25 & 1.626 & -0.20 \\
\hline 4.313 & 8.260 & 23.76 & 39.58 & 2.109 & -0.30 \\
\hline 5.656 & 10.40 & 24.81 & 41.00 & 2.534 & -0.40 \\
\hline 6.919 & 12.53 & 25.71 & 41.85 & 2.914 & -0.50 \\
\hline data $\mathrm{S}_{1}$ & data $\mathrm{S}_{2}$ & data $\mathrm{P}_{1}$ & data $\mathrm{P}_{2}$ & data $V$ & \\
\hline
\end{tabular}

${ }^{a}$ Note that inspection of the nonrelativistic limit reveals that our coupling, $v$, is related by $v=g^{2} /(4 \pi)$ to the coupling $g$ used in Refs. [5 7$]$.

\section{B. Additional complications in the case of fermionic bound-state constituents}

In their numerical studies, Carbonell and Karmanov find, at least for both scalar- and vector-boson exchanges, that beyond some critical value $g_{\mathrm{c}}$ of the respective coupling constant $g$, namely, $g_{\mathrm{c}}=2 \pi$ in the scalar-boson case and $g_{\mathrm{c}}=\pi$ in the vector-boson case, the resulting energy spectrum is unbounded from below. This - strange but for good reasons beyond doubt highly unwanted - feature is cured by introduction of a vertex form factor $F(k), k \equiv p-q$, of the form

$$
F(k)=\frac{\mu^{2}-\Lambda^{2}}{k^{2}-\Lambda^{2}+\mathrm{i} \varepsilon}, \quad \lim _{\Lambda \rightarrow \infty} F(k)=1,
$$

and by regularization of the relevant interaction vertices by replacing the corresponding coupling constant $g$ by $g F(k)$. Moreover, the interaction kernels exhibit a discontinuous behaviour as functions of the respective integration variables. These discontinuities are smoothed by multiplying the entire integral equation, that is, both LHS and RHS, by a factor $\eta(p, P)$ which is reminiscent of the product of the free propagators of two spinless particles carrying momenta $p_{1}$ and $p_{2}$ :

$$
\eta(p, P)=\frac{m^{2}-L^{2}}{\left(\frac{1}{2} P+p\right)^{2}-L^{2}+\mathrm{i} \varepsilon} \frac{m^{2}-L^{2}}{\left(\frac{1}{2} P-p\right)^{2}-L^{2}+\mathrm{i} \varepsilon}, \quad \lim _{L \rightarrow \infty} \eta(p, P)=1, \quad L>m .
$$

Carbonell and Karmanov claim that the latter modification does not change the resulting solutions of the BS equation.

\section{CONSTRUCTION OF EFFECTIVE POTENTIALS BY GEOMETRIC SPECTRAL INVERSION}

We now use our inversion theory [8] to find the potential shape $f(r)$ in the Schrödinger Hamiltonian $H$, Eq. (1), that, for any spectral data set in Table generates those binding energies $E$ for the given values of the coupling parameter $v$. 
Let us start our inversion algorithm, Eq. (17), from a pure Coulomb seed potential $f^{[0]}(r)=-\alpha / r$, with constant $\alpha>0$. Since there are merely ten points for each data set, it is necessary first to represent each energy curve $F(v)$ by a smooth interpolating function. Each data set determines a cutoff value $v_{0}$ of the coupling, defined by $F\left(v_{0}\right)=0$. In cases such as data $\mathrm{P}_{2}$ of Table【, where $v_{0} \approx 30.8$ is large, we found that the inversion algorithm converges very slowly. It became clear that it was much more effective to shift the abscissae of the data by using the new variable $u \equiv v-v_{0}$, in effect inverting $\mathcal{F}(u) \equiv F\left(v_{0}+u\right)$ instead of $F(v)=F\left(v_{0}+u\right)$. Thus, using the data set $\mathrm{P}_{2}$ of Table【, we find the potential shape whose graph is plotted in Fig. 1] an abuse of notation allows us to label the energy ordinate generically as $F(u)$.

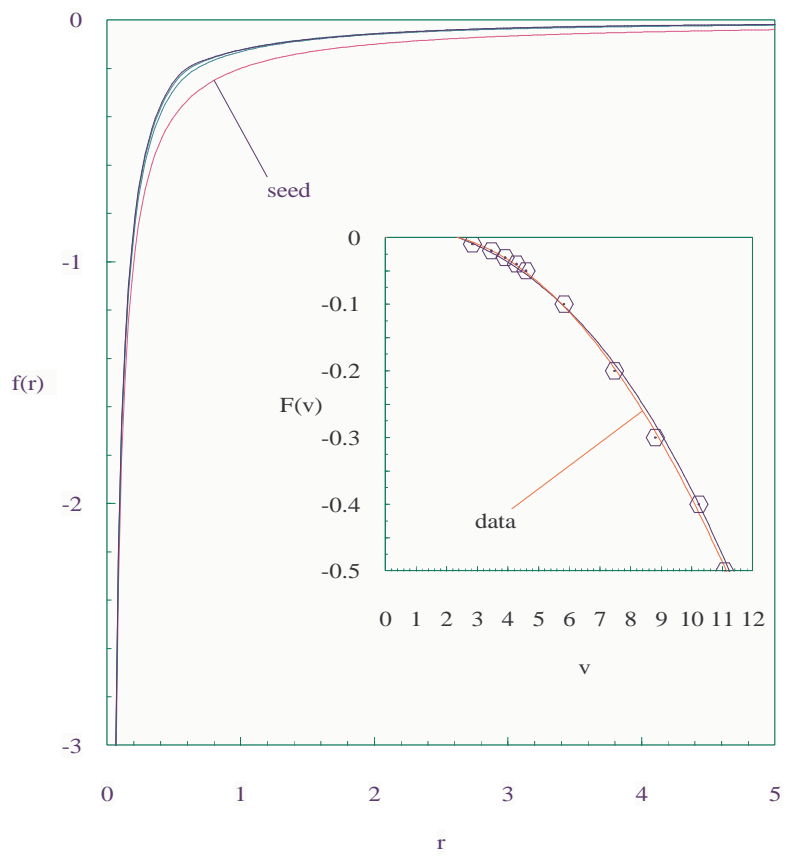

FIG. 1: Geometric inversion of the data set $\mathrm{P}_{2}$ of fermion-antifermion binding energies in Table $\Pi$. We depict, for the potential $f(r)$, a sequence of three iterations of inversion, $f^{[k]}(r), k=0,1,2,3$, where the seed, $f^{[0]}(r)=-0.2 / r$, is the lowest (red) curve, as well as, in the insert graph, both the interpolation curve $F(u)$ of the binding-energy input (hexagons) and the corresponding eigenvalue curve of the Hamiltonian $H=-\Delta+u f^{[3]}(r)$. Since $E=0$ for $v_{0} \approx 30.8$, the sequence inverts $F(u)$, where $u \equiv v-v_{0}$. (Note that $F(u)$ is positive in the interval $u \in\left(0, u_{c}\right), u_{c}=4 b / a^{2}=2.3726$, rising to a maximum of 0.0072 at the interval center.)

\section{MINIMAL SCHRÖDINGER MODELS}

\section{A. Coulomb model}

Encouraged by the effectiveness and rapid convergence of the inversion of Sec. IV] we adopt tentatively the following simplified method. Consider the Schrödinger Hamiltonian $H$ for the relative energy of two particles of common mass $m$,

$$
H=-\frac{1}{m} \Delta+\left(v-v_{0}\right)\left(-\frac{a}{r}+b\right)
$$

where the three adjustable parameters $\left\{a, b, v_{0}\right\}$ are the Coulomb weight $a$, a potential shift $b$, and the critical coupling for vanishing energy, $v_{0}$. All corresponding exact eigenenergies $E_{n \ell}(v)$ are immediately given by the elementary formula

$$
E_{n \ell}(v)=b\left(v-v_{0}\right)-\frac{m a^{2}\left(v-v_{0}\right)^{2}}{4(1+n+\ell)^{2}}, \quad n=0,1,2, \ldots, \quad \ell=0,1,2, \ldots .
$$

For the case at hand, we have $m=1$ for the mass, we set $u \equiv v-v_{0}$, and we consider the ground state (identified by the quantum numbers $n=\ell=0$ ) for which we obtain the energy formula $E=F(u)$ and its exact inversion $f(r)$ as follows:

$$
F(u)=-\frac{a^{2} u^{2}}{4}+b u \quad \longrightarrow \quad f(r)=-\frac{a}{r}+b .
$$


The idea is that we fit the energy formula to the given energy data by finding the best parameter triple $\left\{a, b, v_{0}\right\}$ : this, in turn, specifies the associated potential shape, $f(r)$. The fast convergence of the inversion algorithm that we exhibited numerically in Fig. 1 is, in fact, realized analytically in just a single step, as we now show in the following subsection.

\section{B. Exact inversion in one step}

We prove, for the inversion algorithm of Sec. [II that if the seed is of Coulombic shape, $f^{[0]}(r)=-\alpha / r$, then the first iteration of the algorithm yields $f^{[1]}(r)=-a / r+b$. To show this, we simply apply the inversion algorithm, as follows:

$$
\begin{aligned}
H^{[0]}=-\Delta-\frac{\alpha u}{r} & \Longrightarrow \quad F^{[0]}(u)=-\frac{(\alpha u)^{2}}{4} \\
& \longrightarrow \quad K^{[0]}(r)=\max _{u>0}\left[F^{[0]}(u)-u f^{[0]}(r)\right]=\frac{1}{r^{2}} \\
& \longrightarrow \quad f^{[1]}(r)=\max _{u>0}\left[\frac{F(u)}{u}-\frac{K^{[0]}(r)}{u}\right] \\
& \longrightarrow \quad f^{[1]}(r)=\max _{u>0}\left(-\frac{a^{2} u}{4}-\frac{1}{r^{2} u}+b\right)=-\frac{a}{r}+b .
\end{aligned}
$$

Thus, the inversion $F \rightarrow f$ is achieved in one step. This finding itself is perhaps neither profound nor surprising, but it illuminates our observation, made in the present context, that geometric spectral inversion is very efficient when there exists a critical coupling $v_{0} \neq 0$ and the energy is considered to be a function of $\left(v-v_{0}\right)$. We note in passing that the second algorithmic step yields the $K$-function $K^{[0]}(r)=1 / r^{2}$, which does not depend on the parameter $\alpha$, even though the seed, $f^{[0]}(r)=-\alpha / r$, does. This is consistent with the general invariance of $K$-functions, noted in Eq. (9) above.

\section{Application of the Coulomb model to Bethe-Salpeter binding energies}

We apply the model (23) to each of the data sets in Table【. The fitted values of the parameters $\left\{a, b, v_{0}\right\}$ are collected in Table【whereas the graphical results for the potential shapes $f(r)$ and the corresponding $v$-form energy curves $F(v)$ are depicted in Figs. 2 through 6, the interpolated hexagons represent the original discrete spectral data from Table 1 .

TABLE II: Values found for the parameters $\left\{a, b, v_{0}\right\}$ of the shifted Coulomb potential for each of the five data sets in Table $\square$

\begin{tabular}{cccr}
\hline \hline Data set & $a$ & \multicolumn{1}{c}{$b$} & \multicolumn{1}{c}{$v_{0}$} \\
\hline $\mathrm{S}_{1}$ & 0.0654431 & -0.075782 & 0.539623 \\
$\mathrm{~S}_{2}$ & 0.0233604 & -0.046011 & 1.948014 \\
$\mathrm{P}_{1}$ & 0.1589268 & 0.0 & 16.841319 \\
$\mathrm{P}_{2}$ & 0.1432232 & 0.012167 & 30.76632 \\
$\mathrm{~V}$ & 0.3773324 & -0.086117 & 0.181089 \\
\hline \hline
\end{tabular}

\section{Equivalent Hulthén model}

As is rather well known [24, 25], for the (nonrelativistic) Schrödinger Hamiltonian operator with Hulthén potential,

$$
H=-\Delta+v\left(-\frac{\alpha}{\exp (\beta r)-1}\right), \quad \alpha>0, \quad \beta>0,
$$

the energy eigenvalues of bound states with vanishing orbital angular momentum ( $\ell=0, s$-states) can be given exactly:

$$
E_{n}=-\frac{\left[v \alpha-\beta^{2}(n+1)^{2}\right]^{2}}{[2 \beta(n+1)]^{2}}, \quad v \alpha \geq \beta^{2}(n+1)^{2}, \quad n=0,1,2, \ldots
$$




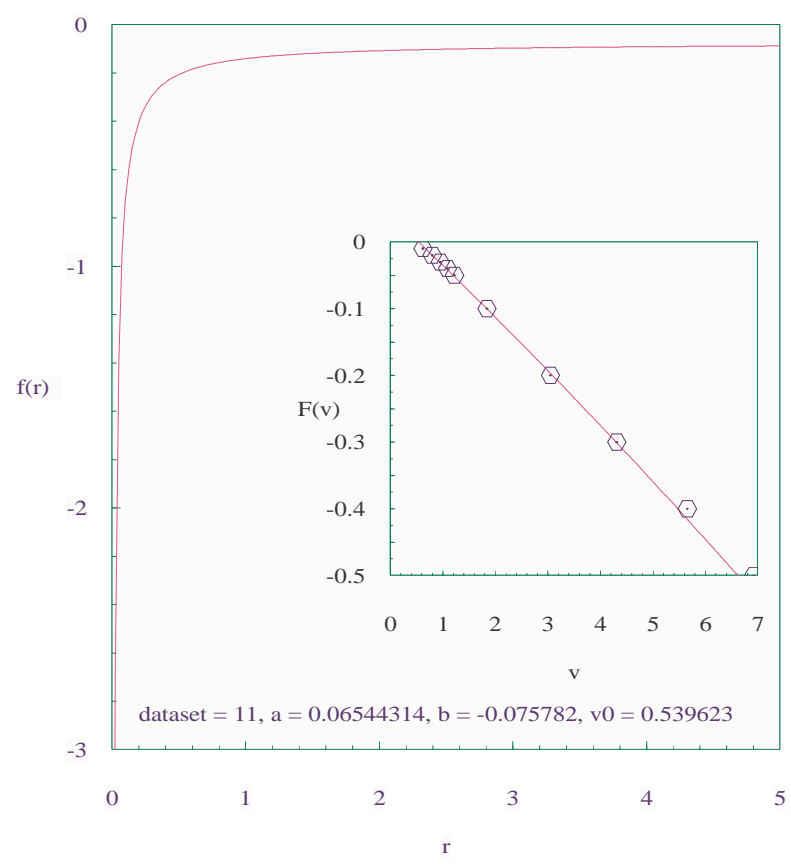

FIG. 2: Binding-energy data $\mathrm{S}_{1}$ from Table凹(interpolated hexagons) and corresponding potential shape given by the model (23).

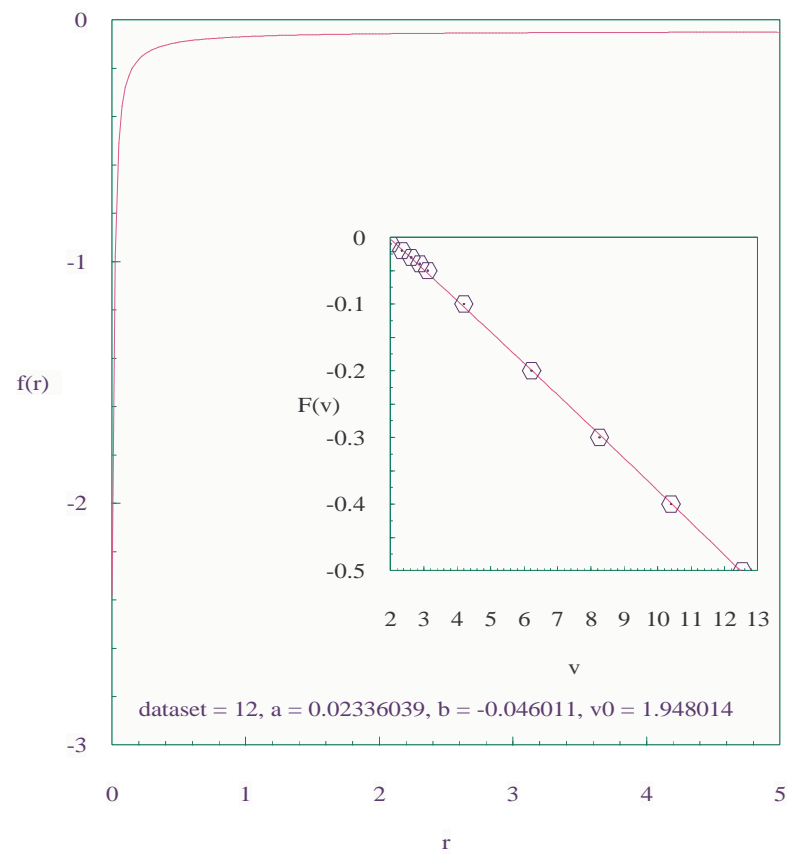

FIG. 3: Binding-energy data $\mathrm{S}_{2}$ from Table $\rrbracket$ (interpolated hexagons) and corresponding potential shape given by the model (23). 


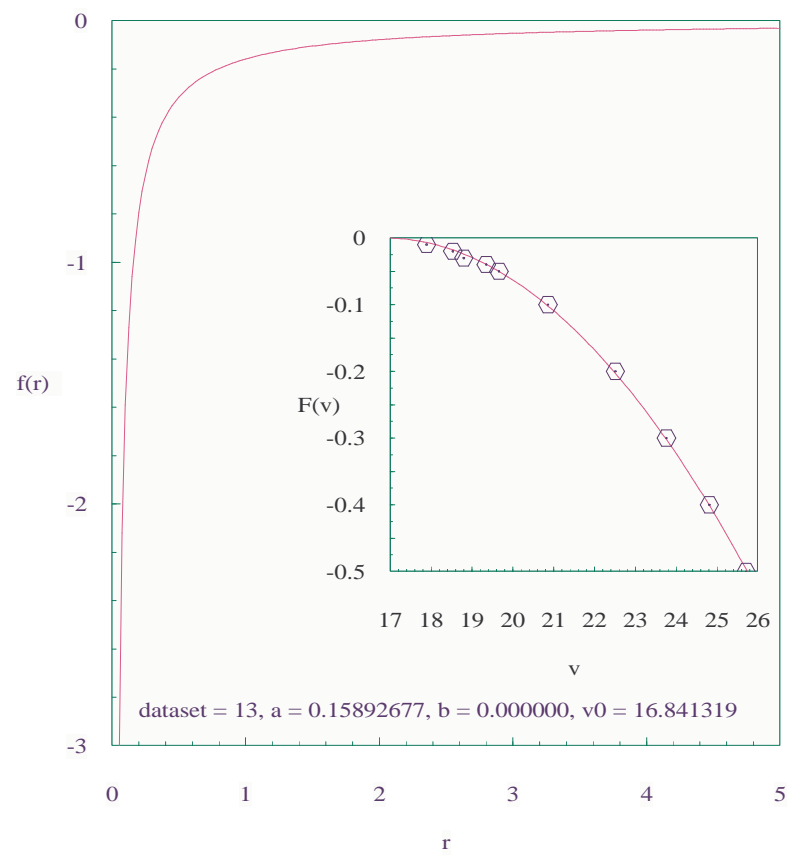

FIG. 4: Binding-energy data $\mathrm{P}_{1}$ from Table】(interpolated hexagons) and corresponding potential shape given by the model (23).

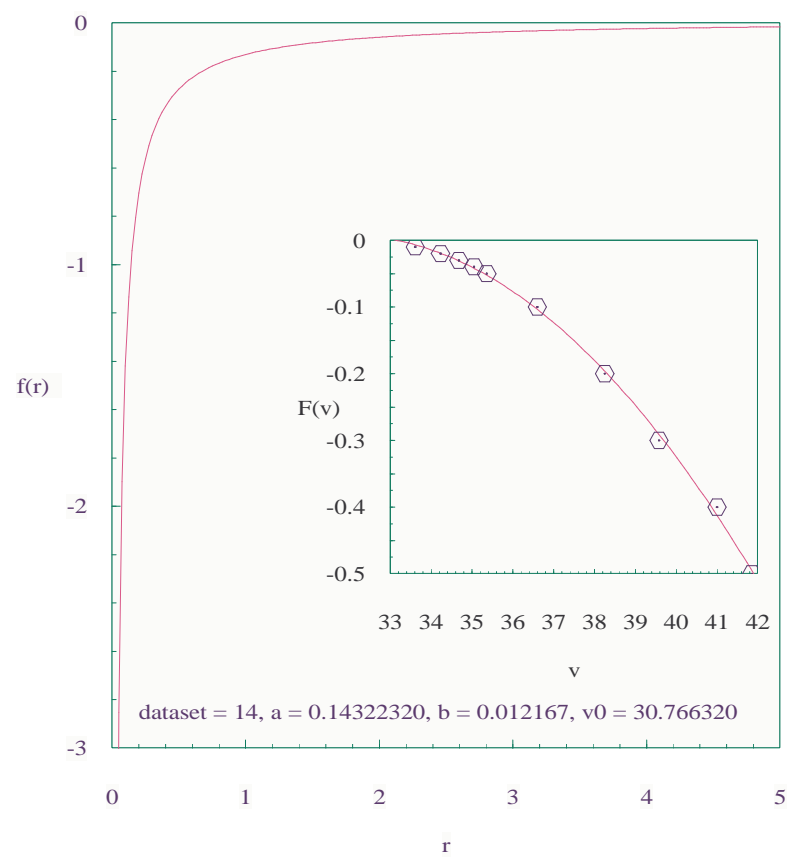

FIG. 5: Binding-energy data $\mathrm{P}_{2}$ from Table凹(interpolated hexagons) and corresponding potential shape given by the model (23). 


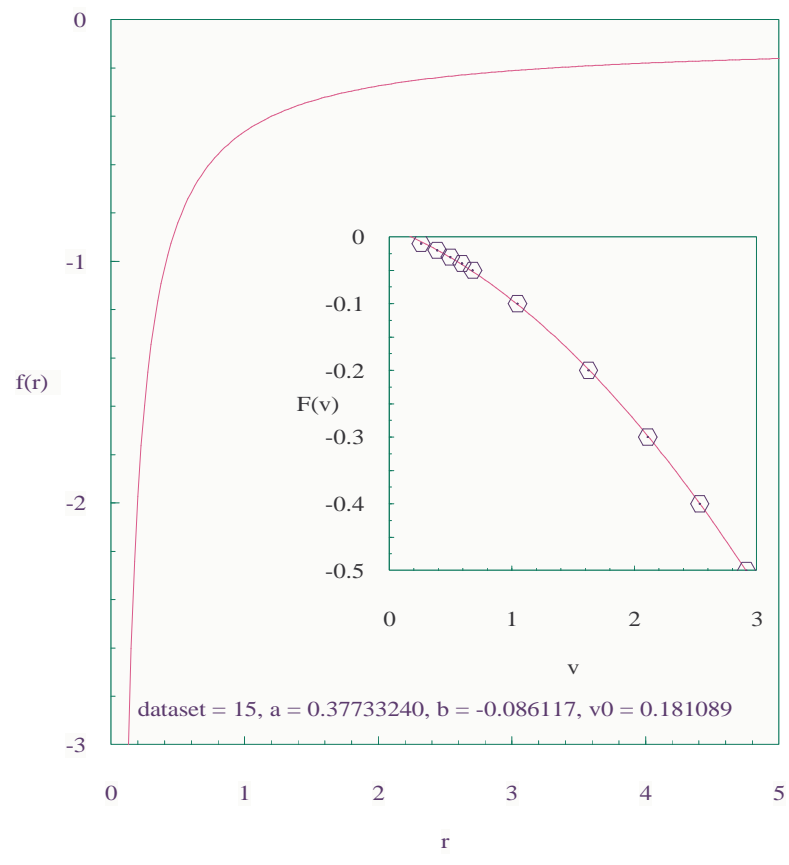

FIG. 6: Binding-energy data V from Table (interpolated hexagons) and corresponding potential shape given by the model (23).

If we consider the ground state $(n=0)$ and set $\alpha=v_{0} a^{2}$ and $\beta=v_{0} a$, then we arrive at the operator-eigenvalue pair

$$
-\Delta+v\left(-\frac{v_{0} a^{2}}{\exp \left(v_{0} a r\right)-1}\right)+b\left(v-v_{0}\right) \quad \longrightarrow \quad E=-\frac{a^{2}\left(v-v_{0}\right)^{2}}{4}+b\left(v-v_{0}\right) .
$$

We note that Eqs. (26) and (23) describe the same spectral curve. There is, however, a qualitative formal difference: in the Coulomb model (23), the potential shape $f(r)$ is simply multiplied by the coupling $\left(v-v_{0}\right)$, whereas, in the Hulthén Hamiltonian (26), the operator term $b\left(v-v_{0}\right)$ contributes the part $b v$ to the potential and simultaneously an additional part $b v_{0}$ is subtracted from the energy operator at the end. Thus, although the Hulthén model involves a pair potential that is similar to the Yukawa potential, its use in the context of the present model structure - where $\left(v-v_{0}\right) f(r)$ so effectively leads to $F(v)$ - does not remain our first choice. Of course, when $b=0$, this difference between the models is removed, and on (understandable) grounds of familiarity in the use of coupling, one might prefer the Hulthén option. In any case, both models are always available and for the ground-state energy they may be considered to be equivalent.

\section{CONCLUSION}

Numerical solutions [5 7 ] to the Bethe-Salpeter equation describing bound states of two fermions yield data for the binding energy $E=F(v)$ as a function of the coupling parameter $v>0$. The form of the data suggests that they may be generated (approximately) by a suitable nonrelativistic model. Meanwhile, we have at our disposal a geometric spectral inversion theory [4, 8 14] which, if $E=F(v)$ is the lowest eigenvalue of the Schrödinger Hamiltonian $H \equiv-\Delta+v f(r)$, reconstructs from the given spectral curve $F(v)$ the underlying potential shape $f(r)$. By first analyzing the fermion data expressed in this manner, as we had done earlier [4] for interacting bosons, we eventually made an elementary discovery: namely, when there exists a nonzero critical value $v_{0}$ of the coupling $v$ and the model Hamiltonian is written in the form $H=-\Delta+\left(v-v_{0}\right) f(r)$, then the spectral data $F(v)$ found for the Bethe-Salpeter two-fermion problem are accurately represented as the eigenvalues of that Hamiltonian $H$ for which the potential shape has, for $a>0$, the elementary form $f(r)=-a / r+b$. As more eigenvalue data become available, we expect to be able to translate the essential features of such relativistic two-particle problems into values for the parameters $\left\{a, b, v_{0}\right\}$ of this minimal nonrelativistic model. 


\section{Acknowledgments}

One of us (RLH) gratefully acknowledges both partial financial support of this work under Grant No. GP3438 from the Natural Sciences and Engineering Research Council of Canada and the hospitality of the Institute for High Energy Physics of the Austrian Academy of Sciences, Vienna, where part of this work was done.

[1] H. A. Bethe and E. E. Salpeter, Phys. Rev. 82 (1951) 309.

[2] M. Gell-Mann and F. Low, Phys. Rev. 84 (1951) 350.

[3] E. E. Salpeter and H. A. Bethe, Phys. Rev. 84 (1951) 1232.

[4] R. L. Hall and W. Lucha, Phys. Rev. D 85 (2012) 125006, arXiv:1205.1447 [math-ph].

[5] J. Carbonell and V. A. Karmanov, PoS LC2010 (2010) 014, arXiv:1009.4522 [hep-ph].

[6] J. Carbonell and V. A. Karmanov, Eur. Phys. J. A 46 (2010) 387, arXiv:1010.4640 [hep-ph].

[7] J. Carbonell and V. A. Karmanov, Few Body Syst. 49 (2011) 205, arXiv:1012.0246 [hep-ph].

[8] R. L. Hall and W. Lucha, J. Math. Phys. 52 (2011) 112102, arXiv:1111.1159 [math-ph].

[9] R. L. Hall, Phys. Rev. A 50 (1994) 2876.

[10] R. L. Hall, J. Phys. A: Math. Gen. 28 (1995) 1771.

[11] R. L. Hall, Phys. Rev. A 51 (1995) 1787.

[12] R. L. Hall, J. Math. Phys. 40 (1999) 699.

[13] R. L. Hall, J. Math. Phys. 40 (1999) 2254.

[14] R. L. Hall, Phys. Lett. A 265 (2000) 28.

[15] W. Lucha, F. F. Schöberl, and D. Gromes, Phys. Rep. 200 (1991) 127.

[16] W. Lucha and F. F. Schöberl, Int. J. Mod. Phys. A 7 (1992) 6431.

[17] W. Lucha and F. F. Schöberl, Int. J. Mod. Phys. A 14 (1999) 2309, arXiv:hep-ph/9812368.

[18] W. Lucha and F. F. Schöberl, Fizika B 8 (1999) 193, arXiv:hep-ph/9812526.

[19] S. J. Gustafson and I. M. Sigal, Mathematical Concepts of Quantum Mechanics (Springer, New York, 2006). [The operator inequality is proved for dimensions $d \geq 3$ on p. 32.]

[20] M. Reed and B. Simon, Methods of Modern Mathematical Physics II: Fourier Analysis, Self-Adjointness (Academic Press, New York, 1975). [The operator inequality is proved on p. 169.]

[21] M. Reed and B. Simon, Methods of Modern Mathematical Physics IV: Analysis of Operators (Academic Press, New York, 1975).

[22] I. M. Gelfand and S. V. Fomin, Calculus of Variations (Prentice-Hall, Englewood Cliffs, 1963. [Legendre transformations are discussed on p. 72.]

[23] W. Feller, An Introduction to Probability Theory and its Applications, Volume II (John Wiley, New York, 1971). [Jensen's inequality is proved on p. 153.]

[24] S. Flügge, Practical Quantum Mechanics (Springer, New York, 1974). [The Hulthén potential is discussed on p. 175.]

[25] R. L. Hall, J. Phys. A: Math. Gen. 25 (1992) 1373. 